\title{
Desgaste profesional en residentes colombianos de cirugía: resultados de un estudio nacional
}

\author{
Luis Carlos Domínguez ${ }^{1}$, Álvaro Sanabria ${ }^{2}$, Adonis Ramírez ${ }^{3}$, Felipe Vargas ${ }^{4}$, \\ Maikel Pacheco ${ }^{5}$, Germán JimÉneZ 6
}

Palabras clave: agotamiento profesional; cirugía general; educación médica; condiciones de trabajo.

\section{Resumen}

Objetivo. El sindrome de desgaste profesional es una entidad muy prevalente en cirujanos que tiene un impacto negativo en los resultados clínicos y educativos. El objetivo del estudio fue determinar la prevalencia del sindrome de desgaste profesional en residentes colombianos de cirugía, y su variación en función de la edad, el sexo, el tipo de programa, el año de residencia y la región geográfica.

Métodos. Se utilizó el Maslach Burnout InventoryHuman Services Survey (MBI-HSS) en 202 residentes.

1 Médico cirujano, MHPE, Departamento de Cirugía, Universidad de la Sabana, Chía, Colombia

2 Médico cirujano, MSc, PhD, Departamento de Cirugía, Universidad de Antioquia, Medellín, Colombia

3 Médico cirujano, MEd, Departamento de Cirugía, Universidad Surcolombiana, Neiva, Colombia

4 Médico cirujano, Departamento de Cirugía, Universidad del Rosario, Bogotá, D.C., Colombia

5 Médico cirujano, Departamento de Cirugía, Fundación Universitaria Ciencias de la Salud, Bogotá, D.C., Colombia

6 Médico cirujano, Departamento de Cirugía, Fundación Universitaria Sanitas, Bogotá, D.C., Colombia

Fecha de recibido: 14 de diciembre de 2016

Fecha de aprobación: 7 de abril de 2017

Citar como: Domínguez LD, Sanabria Á, Ramírez A, Vargas F, Pacheco M, Jiménez G. Desgaste profesional en residentes de cirugía colombianos: resultados de un estudio nacional. Rev Colomb Cir. 2017;32:121-27.
Se calculó el porcentaje global de sindrome de desgaste profesional. Se realizaron análisis univariados para el puntaje global del MBI-HSS y sus subescalas en función de los factores propuestos, y multivariado para evaluar su independencia.

Resultados. La prevalencia del síndrome de desgaste profesional fue de 33,15\%. No se encontraron diferencias significativas entre el puntaje global y el de las subescalas del MBI-HSS entre residentes con sindrome de desgaste profesional y sin él, de acuerdo con las variables propuestas. La prevalencia del síndrome de desgaste profesional fue mayor en los programas del centro del país $\left(O R=4,76 ; C_{95 \%} 2,0-11,2\right)$.

Conclusiones. El síndrome de desgaste profesional en residentes colombianos de cirugía es una entidad prevalente que no se relaciona con la edad, el sexo, el tipo de programa ni el año de residencia. Existen variaciones regionales que indican un mayor riesgo de síndrome de desgaste profesional entre los residentes de los programas del centro del país, las cuales deben explorarse en profundidad.

\section{Introducción}

El aprendizaje en las residencias médicas es experiencial y se basa principalmente en el trabajo clínico. El 'sentirse bien' en el ambiente de trabajo es fundamental para el buen desempeño profesional del residente, en aspectos asistenciales y educativos, y para los pacientes, en términos del cuidado de la salud y la seguridad ${ }^{1,2}$. No obstante, la inadecuada estructura del ambiente de trabajo es el principal determinante del pobre bien- 
estar, el cual se manifiesta en los residentes al sentirse 'quemados' o abatidos (burned out) o 'no enganchados' al trabajo ${ }^{3}$. El problema es que estar 'quemado' se asocia con disminución en el rendimiento, ausentismo, deserción y problemas de salud física y psicológica para el residente, y también, con mayores errores médicos, deshumanización en la atención y complicaciones para los pacientes

En particular, se sabe que la estructura del ambiente de trabajo en cirugía es muy demandante en aspectos como carga, presión asistencial y horas de trabajo, y que por estas razones existe un elevado número de residentes de cirugía 'quemados', quienes ven afectados su estilo y calidad de vida en diferentes contextos ${ }^{4-6}$. Por ejemplo, en varios estudios recientes se reporta que el $69 \%$ de los residentes de cirugía en los Estados Unidos, el $64 \%$ en Canadá y el 57,9\% en países de ingreso bajo a medio, entre otros, se encuentran 'quemados' por estos motivos ${ }^{4,7,8}$. Igualmente, se sabe que este fenómeno tiene que ver con los acelerados cambios en los sistemas educativos y sanitarios que han introducido transformaciones importantes en el ejercicio profesional, especialmente en los últimos cinco años ${ }^{6}$.

En este contexto, aunque es urgente la investigación sobre el efecto de las políticas de restricción en el número de horas semanales de trabajo y el riesgo de 'quemarse' en el trabajo, hasta el momento, pocos estudios realizados en Norteamérica indican que la prevalencia del problema se ha reducido, pues, aunque actualmente los residentes trabajan menos horas, la carga de trabajo y la presión asistencial han aumentado ${ }^{6,9}$. Últimamente, las consecuencias específicas para los residentes de cirugía y para los pacientes tienen que ver con el mayor número de problemas físicos y psicológicos, incluyendo mayor propensión al alcoholismo e ideación suicida, y con el aumento en la tasa de errores y las complicaciones, respectivamente ${ }^{6}$.

La información disponible indica que existen estrategias enfocadas en el individuo y otras estructurales enfocadas en las organizaciones, que pueden resultar en la reducción clínicamente significativa de este problema entre los médicos ${ }^{10}$. Sin embargo, lo que aún requiere investigación es la efectividad de estas intervenciones, así como la forma de prevenirlo ${ }^{6}$. Un llamado inicial es el de conocer la prevalencia del problema en contextos específicos, para luego enfrentarlo integralmente ${ }^{10}$.
En Colombia, no existe información sobre la prevalencia del problema entre residentes de cirugía, teniendo en cuenta la dinámica actual del sistema sanitario y su potencial impacto en la educación de posgrado. El conocer el estado del problema en el país puede aclarar aspectos relacionados con el bienestar en el trabajo en cirugía, proporcionar evidencia para la identificación de un problema latente, y orientar a su futura intervención y seguimiento.

El objetivo del estudio fue determinar la prevalencia del síndrome de desgaste profesional en residentes colombianos de cirugía, y su variación en función de la edad, el sexo, el tipo de programa, el año de residencia y la región geográfica.

\section{Métodos}

\section{Población}

Este es un estudio analítico de tipo transversal, hecho en Colombia. El estudio fue aprobado en sus aspectos éticos por la Comisión de Educación Médica de la Facultad de Medicina, Universidad de la Sabana (Colombia). Se invitaron a participar a los integrantes de los 21 programas de especialización en Cirugía General disponibles en Colombia (2015). Se estima una población cercana a 400 residentes activos para el 2015. Para este propósito, se contactaron a los directores de programa o a los decanos de las Facultades de Medicina. Del total, cinco programas no respondieron a la invitación y uno decidió voluntariamente no participar. Al final, participaron 15 programas ubicados en siete ciudades, que incluían una población aproximada de 284 residentes. Estos residentes fueron invitados a diligenciar una cuestionario de forma voluntaria, anónima y confidencial (tanto individual como institucionalmente).

\section{Mediciones y manejo de la información}

El estar 'quemado' en el trabajo se puede evaluar objetivamente mediante la medición del síndrome de desgaste profesional (burnout syndrome). Este síndrome representa un estado negativo de bienestar, resultante del cansancio emocional, la despersonalización y la falta de realización personal en el trabajo ${ }^{11}$. El cansancio emocional se refiere a la sensación de estar emocionalmente agotado por el contacto propio con otras personas, y es la dimensión central del síndrome de desgaste profesional ${ }^{12}$. La despersonalización se refiere a una reacción negativa o 
excesivamente individual hacia estas otras personas, que son los destinatarios de servicios o atención. Por último, la poca realización personal se refiere a una disminución de los propios sentimientos de competencia y el logro de éxito en el trabajo.

En el presente estudio se midió el síndrome de desgaste profesional mediante el instrumento Maslach Burnout Inventory-Human Services Survey (MBI-HSS) disponible en español ${ }^{13}$. La escala está compuesta por 22 ítems que miden las tres dimensiones del síndrome de desgaste profesional, los cuales se califican individualmente mediante una escala de Likert que varía desde 0 (nunca) hasta 6 (siempre o cada día). Estos son algunos ejemplos de los ítems: "Siento que trato con mucha efectividad los problemas de mis pacientes", "me siento frustrado por el trabajo", "siento que estoy tratando a algunos pacientes como si fuesen objetos impersonales". La confiabilidad reportada del MBI-HSS en español es $\alpha=0,74{ }^{14}$. Los aspectos relacionados con el MBI-HSS se encuentran descritos en la tabla 1. Además, se obtuvo información general de los participantes sobre sexo, edad, tipo de programa (público o privado), año de residencia y localización geográfica del programa.

La información fue recolectada mediante un cuestionario en papel, entre octubre y diciembre del 2015. Los investigadores principales del estudio recolectaron directamente la información en 11 programas. En los programas restantes fue recolectada directamente por los directores de programa quienes retornaron los cuestionarios a los investigadores principales.

La información fue almacenada en una base de datos. Una vez completados los resultados del estudio, los de cada programa fueron enviados a cada uno de los directores de programa o a los decanos, para su análisis interno. Por razones de confidencialidad, en este estudio se omitieron los nombres de los programas y se agrupó la información, para uno de sus análisis, en tres zonas geográficas del país (Centro, Occidente y Caribe).

\section{Análisis estadístico}

Se calcularon las estadísticas descriptivas de la población en frecuencias y medias. Para cada una de las subescalas del MBI-HSS, se calcularon la media, las desviaciones estándar y los rangos mediante el cálculo de las puntuaciones por ítem. Posteriormente, se calculó el porcentaje global del síndrome de desgaste profesional de acuerdo con las recomendaciones estándar del MBI-HSS (tabla 1). Se llevaron a cabo análisis univariados para cada una de las puntuaciones de las subescalas ajustadas por sexo, año de residencia y tipo de programa. Finalmente, se hicieron comparaciones entre los individuos con síndrome de desgaste profesional y sin él, en función de edad, sexo, tipo de programa (público o privado), año de residencia y zona geográfica del programa. Para estos análisis se utilizaron las estimaciones de las pruebas t de Stdent y ji al cuadrado, respectivamente (significativo si $\mathrm{p}<0,05$ ). Para evaluar la independencia de los factores, se hizo un análisis de regresión logística con las variables sexo, edad, tipo de institución, año de residencia y región. Para la variable 'región', se crearon variables indicadoras. Para el análisis, se utilizó el programa Stata $13^{\circledR}$.

\section{Resultados}

Del total de la población, 202 residentes $(71,6 \%)$ respondieron el cuestionario MBI-HSS. La edad promedio de los participantes fue de 28,63 $\pm 2,96$ (rango: 23 a 41) años. En relación con las subescalas, se encontraron niveles altos de cansancio emocional en el 55,4\% de los residentes, altos niveles de despersonalización en el 30,2 \%, y bajos niveles de realización personal en el $41,6 \%$. La distribución porcentual de las subescalas del MBI-HSS se presenta en la figura 1.

El porcentaje global de residentes que cumplieron con el criterio de síndrome de desgaste profesional fue de $33,15 \%$ (67 residentes). No se encontraron diferencias significativas entre residentes con síndrome de desgaste

TABLA 1.

Descripción del Maslach Burnout Inventory-Human Services Survey

\begin{tabular}{lcccc}
\hline \multirow{2}{*}{\multicolumn{1}{c}{ Subescala }} & \multicolumn{4}{c}{ Puntaje } \\
\cline { 2 - 5 } & Alto & Moderado & Bajo & \\
\hline Cansancio emocional & $\geq 27$ & 17 a 26 & 0 a 16 & 0 a $6 *$ \\
\hline Despersonalización & $\geq 14$ & 9 a 13 & 0 a 8 & 0 a 6 \\
\hline Realización personal** & 0 a 30 & 31 a 36 & $\geq 37$ & 0 a 6 \\
\hline
\end{tabular}

Síndrome de desgaste profesional = cansancio emocional alto más despersonalización alta más realización personal baja

* Donde 0 es nunca y, 6 , es todos los días.

** Se interpreta de forma opuesta a cansancio emocional y despersonalización. 


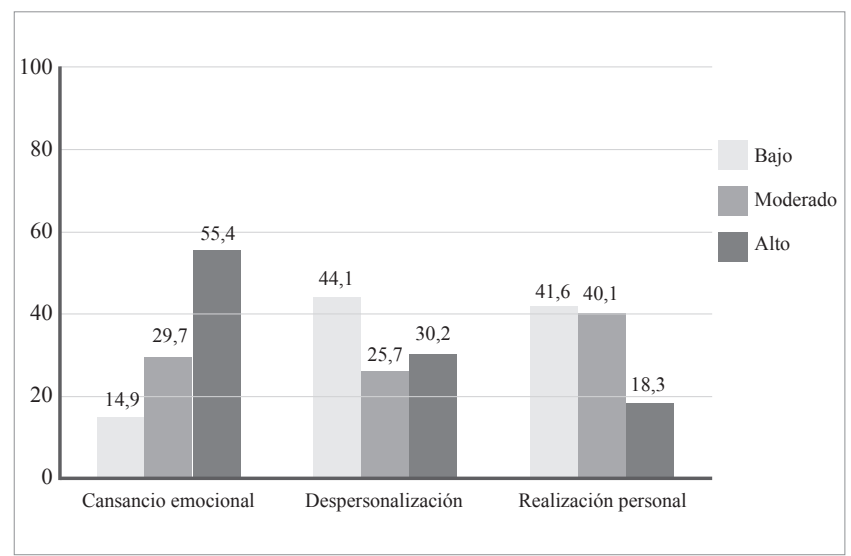

Figura 1. Porcentajes en las subescalas del sindrome de desgaste profesional en residentes de Cirugía General en Colombia, según el Maslach Burnout Inventory-Human Services Survey (MBI-HSS)

profesional y sin él según la edad, el sexo, el tipo de programa (público o privado) o el año de residencia. La prevalencia del síndrome de desgaste profesional fue mayor en los programas del centro del país, en comparación con los de Occidente y la región Caribe $(p<0,001)$. Las características de la población asociadas con el síndrome de desgaste profesional están resumidas en la tabla 2.

No se encontraron diferencias significativas en las subescalas de cansancio emocional y despersonalización según el sexo o el año de residencia. Se encontraron diferencias entre los puntajes de todas las subescalas según la región geográfica, siendo mayor el desgaste emocional y la despersonalización, y menor la realización personal, en los programas del centro del país en comparación con los del Occidente y el Caribe $(\mathrm{p}<0,05)$. Otras características de las subescalas del MBI-HSS según sexo, tipo de programa y año de residencia están resumidas en la tabla 3. En el análisis de la regresión logística, se encontró que la variable 'región Centro' fue la única variable independiente con asociación estadísticamente significativa con el síndrome de desgaste profesional $\left(\mathrm{OR}=4,76 ; \mathrm{IC}_{95 \%} 2,0-11,2\right)$.

\section{Discusión}

El objetivo de este estudio fue evaluar la prevalencia del síndrome de desgaste profesional en residentes colombianos de cirugía según el MBI-HSS. En primer lugar, se identificó que uno de cada tres residentes estaba 'quemado' en el trabajo al momento de la medición. Esta prevalencia es menor a la reportada en otros países,
TABLA 2

Características de la población asociadas con el síndrome de desgaste profesional en análisis univariados

\begin{tabular}{|c|c|c|c|c|}
\hline & Total & $\begin{array}{c}\text { Sin síndrome } \\
\text { de desgaste } \\
\text { profesional } \\
\text { n \% }\end{array}$ & $\begin{array}{c}\text { Con } \\
\text { síndrome } \\
\text { de desgaste } \\
\text { profesional } \\
\text { n \% } \\
\end{array}$ & $\mathbf{p}$ \\
\hline Residentes & 202 & $135(66,83)$ & $67(33,17)$ & \\
\hline Sexo & & & & ND \\
\hline Masculino & 129 & $83(64,34)$ & $46(35,66)$ & \\
\hline Femenino & 73 & $52(71,23)$ & $21(28,77)$ & \\
\hline Tipo de programa & & & & ND \\
\hline Público & 71 & $46(64,49)$ & $25(35,21)$ & \\
\hline Privado & 131 & $89(67,94)$ & $42(32,06)$ & \\
\hline Nivel de residencia & & & & ND \\
\hline 1 & 67 & $44(65,67)$ & $23(34,33)$ & \\
\hline 2 & 43 & $28(65,12)$ & $15(34,88)$ & \\
\hline 3 & 45 & $31(68,89)$ & $14(31,11)$ & \\
\hline 4 & 47 & $32(68,09)$ & $15(31,91)$ & \\
\hline $\begin{array}{l}\text { Distribución } \\
\text { geográfica }\end{array}$ & & & & $<0,001$ \\
\hline Centro & 114 & $64(56,14)$ & $50(43,86)$ & \\
\hline Occidente & 53 & $44(83,02)$ & $9(16,98)$ & \\
\hline Caribe & 35 & $27(77,14)$ & $8(22,86)$ & \\
\hline
\end{tabular}

ND; no hay diferencia

cercana al $60 \%{ }^{4,5}$, pero es alta en términos absolutos, en particular, si se acepta la asociación entre el síndrome de desgaste profesional y los resultados negativos para los pacientes y el personal sanitario en cirugía ${ }^{15}$. Además, como se ha indicado en otros estudios ${ }^{16}$, la proporción del síndrome de desgaste profesional es idéntica en los cirujanos graduados, hallazgo que podría indicar su cronicidad aun después de graduarse.

Un segundo resultado de este estudio tiene que ver con que el síndrome de desgaste profesional no varió en función de la edad, el sexo, el tipo de programa o el año de residencia. En general, se suele asumir que los primeros años de residencia son más exigentes y demandantes, pues se conjuga una desbordante exigencia asistencial y académica. También, se asume que después de la adaptación inicial, el residente transita hacia una etapa de confort, en la cual ya conoce las condiciones 
TABLA 3

Características de las subescalas del Maslach Burnout Inventory-Human Services Survey (MBI-HSS) según sexo, tipo de programa año de residencia y región geográfica (promedios, desviaciones estándar y rangos)

\begin{tabular}{lcrrrr}
\hline \multicolumn{4}{c}{ Sexo } \\
\hline Subescala & Total $(\mathbf{n}=\mathbf{2 0 2})$ & Masculino $(\mathbf{n = 1 2 9})$ & Femenino $(\mathbf{n}=\mathbf{7 3})$ & $\mathbf{p}$ \\
\hline Cansancio emocional & $28,11 \pm 10,23(5-52)$ & $27,27 \pm 9,70(7-50)$ & $29,60 \pm 11,02(5-52)$ & 0,06 \\
\hline Despersonalización & $10,35 \pm 6,36(0-28)$ & $10,91 \pm 6,38(0-27)$ & $9,36 \pm 6,28(0-28)$ & 0,95 \\
\hline Realización personal & $35 \pm 4,81(17-42)$ & $35,07 \pm 4,79(17-42)$ & $34,89 \pm 4,88(21-42)$ & 0,60 \\
\hline
\end{tabular}

Tipo de programa

\begin{tabular}{|c|c|c|c|c|c|c|}
\hline Subescala & Total $(\mathrm{n}=\mathbf{2 0 2})$ & \multicolumn{2}{|c|}{ Privado $(n=131)$} & \multicolumn{2}{|r|}{ Público $(n=71)$} & p \\
\hline Cansancio emocional & $28,11 \pm 10,23(5-52)$ & \multicolumn{2}{|c|}{$27,70 \pm 9,82(5-52)$} & \multicolumn{2}{|c|}{$29,87 \pm 10,99(7-52)$} & 0,07 \\
\hline Despersonalización & $10,35 \pm 6,36(0-28)$ & \multicolumn{2}{|c|}{$10,67 \pm 6,22(0-28)$} & \multicolumn{2}{|c|}{$9-77 \pm 6,64(0-24)$} & 0,83 \\
\hline Realización personal & $35 \pm 4,81(17-42)$ & \multicolumn{2}{|c|}{$34,41 \pm 4,87(17-42)$} & \multicolumn{2}{|c|}{$36,11 \pm 4,52(23-42)$} & $<0,001$ \\
\hline \multicolumn{7}{|l|}{ Nivel de residencia } \\
\hline Subescala & Total $(n=202)$ & \multicolumn{2}{|c|}{ Nivel I-II (n=120) } & \multicolumn{2}{|c|}{ Nivel III-IV (n=92) } & $\mathbf{p}$ \\
\hline Cansancio emocional & $28,11 \pm 10,23(5-52)$ & \multicolumn{2}{|c|}{$28,10 \pm 10,44(5-52)$} & \multicolumn{2}{|c|}{$28,11 \pm 10,04(8-49)$} & 0,49 \\
\hline Despersonalización & $10,35 \pm 6,36(0-28)$ & \multicolumn{2}{|c|}{$10,50 \pm 6,49(0-28)$} & \multicolumn{2}{|c|}{$10,17 \pm 6,26(0-27)$} & 0,64 \\
\hline Realización personal & $35 \pm 4,81(17-42)$ & \multicolumn{2}{|c|}{$34,57 \pm 5,14(17-42)$} & \multicolumn{2}{|c|}{$35,53 \pm 4,36(24-42)$} & 0,07 \\
\hline \multicolumn{7}{|l|}{ Región } \\
\hline Subescala & Total $(n=202)$ & Centro $(n=114)$ & Caribe & 35) & Occidente $(n=53)$ & $\mathbf{p}$ \\
\hline Cansancio emocional & $28,11 \pm 10,23(5-52)$ & $29,4 \pm 9,6(8-52)$ & $24,2 \pm 11$ & $5-49)$ & $27,9 \pm 10,2(9-52)$ & 0,02 \\
\hline Despersonalización & $10,35 \pm 6,36(0-28)$ & $11,7 \pm 6,5(0-28)$ & $8,8 \pm 6$ & $-25)$ & $8,4 \pm 5,1(0-22)$ & 0.0001 \\
\hline Realización personal & $35 \pm 4,81(17-42)$ & $34,1 \pm 4,8(21-42)$ & $37,7 \pm 3$ & $9-42)$ & $32,2 \pm 5,0(17-42)$ & 0,003 \\
\hline
\end{tabular}

y las características de la práctica y posee mejores herramientas para el manejo de su entorno. Nuestros resultados descartan estas hipótesis, ya que la prevalencia del síndrome de desgaste profesional es constante a medida que avanza el programa. De igual forma, el sexo no se relacionó con un mayor efecto sobre el puntaje global del síndrome de desgaste profesional y el de sus subescalas. Aunque se reconoce que la cirugía es una profesión 'masculina' y que puede existir una tendencia según el sexo en la formación que puede favorecer el desgaste en la mujeres ${ }^{17,18}$, los resultados de este estudio descartaron esta asociación, en particular, si se considera que el $28 \%$ de la población evaluada era del sexo femenino. Finalmente, las posibles asociaciones entre la gravedad del síndrome de desgaste profesional y el carácter privado o público de los programas, en razón de los aspectos socioeconómicos, fueron descartadas en este estudio.
Un tercer resultado fue la mayor prevalencia y riesgo de presentar síndrome de desgaste profesional en los programas del centro del país, la cual duplica a la de otras regiones. Es sabido que las culturas de las regiones son diferentes ${ }^{19}$. Esto puede explicarse por los diferentes estilos de educación y práctica profesional a nivel general que podrían advertir sobre las variaciones regionales de la educación de posgrado en Medicina. Sin embargo, el sustentar el efecto cultural sobre las metodologías y los factores involucrados en la educación de posgrado, requiere nuevas investigaciones.

Este estudio tiene fortalezas y debilidades. Es el primer estudio realizado específicamente en residentes de Cirugía General en Colombia y abre la puerta para su seguimiento y futuro estudio. Este estudio incluyó residentes de varias universidades y regiones, y contó con una población representativa de acuerdo con el año 
de residencia. Se utilizaron instrumentos previamente validados en el contexto nacional, lo cual permite que los resultados sean comparables con estudios preliminares. Igualmente, la tasa de respuesta de los participantes fue adecuada $(>70 \%)$, lo cual sustenta los resultados obtenidos. Entre las limitaciones, las cuales están contenidas en el diseño del estudio, se encuentran la ausencia de seguimiento longitudinal y la asociación con otros resultados de interés. En particular, la falta de asociación con otros resultados, como la satisfacción en el trabajo, la calidad de vida, el rendimiento académico y la seguridad del paciente, entre otros, hace que los resultados de este estudio sean solamente una mirada global del problema, sin una determinación de su impacto potencial y de sus consecuencias.

Este estudio tiene implicaciones para la práctica y la investigación futura. En primer lugar, estos resultados aportan información sobre la prevalencia de un problema latente en las universidades y en los programas académicos. La receptividad de los programas de residencia a la retroalimentación individual de los resultados de este estudio, abre oportunidades para el mejoramiento interno de la calidad de la educación y el clima de aprendizaje.

También, abre la puerta para el seguimiento longitudinal del síndrome de desgaste profesional en cirugía en Colombia y ofrece posibilidades para nuevas investigaciones. Una de estas tiene que ver con la asociación entre el síndrome de desgaste profesional y los resultados clínicos en el paciente, y otra sobre la asociación entre el síndrome de desgaste profesional y los resultados educativos en los residentes. En particular, debe explorarse la asociación con el clima de aprendizaje, y el bienestar global en el ambiente de trabajo, mediante estudios que permitan explorar otros estados de bienestar, como el contrato, la apatía y el aburrimiento en el trabajo.

Los resultados también ofrecen la oportunidad de explorar las diferencias en la educación quirúrgica en Colombia a nivel regional, en particular, frente a la asociación que tiene el efecto de la cultura educativa en cirugía con las medidas de desempeño del residente.

Este estudio aporta a la colaboración institucional para la evaluación de medidas comunes de desempeño y de interés nacional, en particular, frente a la crisis actual del recurso humano en salud en Colombia. No obstante, a la fecha no existen estudios que exploren cuáles son los factores desencadenantes en el ambiente de trabajo que expliquen el síndrome de desgaste profesional y que puedan ser modificados por los educadores. Los resultados obligan al diseño de nuevos estudios que permitan identificar dichos factores, y que favorezcan la construcción de herramientas y estrategias que ayuden a disminuir esta prevalencia.

En conclusión, el síndrome de desgaste profesional en residentes colombianos de Cirugía es una entidad altamente prevalente, independiente del sexo, la edad, el año de residencia y el tipo de programa. Existen variaciones regionales que deben explorarse en futuros estudios.

Conflictos de interés: ninguno reportado

\title{
Professional burnout in Colombian surgical residents: results of a national study
}

\begin{abstract}
Objectives: The Burnout Syndrome (BS), a highly prevalent entity among surgeons, holds negative impact on clinical and educational outcomes. Our aim was to measure the prevalence of BS in Colombian surgical residents, and their variation according to age, gender, type of program, level of residence and geographic region.

Methods: The MBI-Human Services Survey (MBI-HSS) was administered to 202 surgical residents. The overall percentage of BS was calculated. Univariate analyses were performed for the global score of the MBI-HSS and its subscales according to the proposed variables, and multivariate analyses were conducted in order to assess their independence.
\end{abstract}


Results: The prevalence of BS was 33.15\%. No significant difference was found in the global and sub-scores of the $M B I-H S S$ between residents with BS and no BS according the proposed variables. The prevalence of BS was higher in the central region of the country (OR 4.76 IC95\% 2.0-11.2).

Conclusions: BS in Colombian surgical residents is a prevalent entity, which is not related to age, gender, and type of program or level of residence. There are regional variations indicating a higher risk of BS among active residents in programs in the central region of the country, which requires further studies.

Key words: Burnout Syndrome, wellbeing, surgery, Colombia, medical education.

\section{Referencias}

1. Boor K, van Der Vleuten C, Teunissen P, Scherpbier A, Scheele F. Development and analysis of D-RECT, an instrument measuring residents' learning climate. Med Teach. 2011;33:820-7.

2. Lases SS, Arah OA, Pierik EG, Heineman E, Lombarts MJ. Residents' engagement and empathy associated with their perception of faculty's teaching performance. World J Surg. 2014;38:2753-60.

3. Dyrbye L, Shanafelt T. A narrative review on burnout experienced by medical students and residents. Med Educ. 2016;50:13249.

4. Elmore LC, Jeffe DB, Jin L, Awad MM, Turnbull IR. National Survey of Burnout among US General Surgery Residents. J Am Coll Surg. 2016;223:440-51.

5. Pulcrano M, Evans SR, Sosin M. Quality of life and burnout rates across surgical specialties: A systematic review. JAMA Surg. 2016;151:970-8.

6. Dimou FM, Eckelbarger D, Riall TS. Surgeon burnout: A systematic review. J Am Coll Surg. 2016;222:1230-9.

7. Malik AA, Bhatti S, Shafiq A, Khan RS, Butt UI, Bilal SM, et al. Burnout among surgical residents in a lower-middle income country - Are we any different? Ann Med Surg (Lond). 2016;9:28-32.

8. Kholdebarin R, Helewa RM, Hochman DJ. Evaluation of a regional acute care surgery service by residents in general surgery. J Surg Educ. 2011;68:290-3.

9. Everett CB, Helmer SD, Osland JS, Smith RS. General surgery resident attrition and the 80-hour workweek. Am J Surg. 2007;194:751-7.

10. West CP, Dyrbye LN, Erwin PJ, Shanafelt TD. Interventions to prevent and reduce physician burnout: A systematic review and meta-analysis. Lancet. 2016; 388:2272-2281.

11. Oerlemans WG, Bakker AB. Burnout and daily recovery: A day reconstruction study. J Occup Health Psychol. 2014;19:303-14.
12. Demerouti E, Mostert K, Bakker AB. Burnout and work engagement: A thorough investigation of the independency of both constructs. J Occup Health Psychol. 2010;15:209-22.

13. Maslach C, Schaufeli WB, Leiter MP. Job burnout. Annu Rev Psychol. 2001;52:397-422.

14. Olivares-Faúndez VE, Mena-Miranda L, Jélvez-Wilke C, MacíaSepúlveda F. Validez factorial del Maslach Burnout Inventory Human Services (MBI-HSS) en profesionales chilenos. Universitas Psychologica. 2014;13:145-59.

15. Shanafelt TD, Balch CM, Bechamps G, Russell T, Dyrbye L, Satele $\mathrm{D}$, et al. Burnout and medical errors among American surgeons. Ann Surg. 2010;251:995-1000.

16. Vega V, Sanabria A, Domínguez L, Osorio C, Ojeda C, Bejarano M. Síndrome de desgaste profesional en cirujanos colombianos. Rev Colomb Cir. 2009;24:153-64.

17. Cortés-Flores AO, Fuentes-Orozco C, López-Ramírez MKL, Velásquez-Ramírez GA, Farías-Llamas OA, Olivares-Becerra $\mathrm{JJ}$, et al. Academic medicine and gender: Women in surgical specialities. Gac Med Mex. 2005;141:341-4.

18. Bruce AN, Battista A, Plankey MW, Johnson LB, Marshall MB. Perceptions of gender-based discrimination during surgical training and practice. Med Educ Online. 2015;20:25923.

19. Díaz-Camacho P. El alma colombiana. Idiosincrasia e identidades culturales en Colombia. Hallazgos. 2012;10:119-41.

Correspondencia: Luis Carlos Domínguez, MD

Correo electrónico:

carlos.dominguez@unisabana.edu.co

Bogotá, D.C. 